\title{
On the impact of a phosphoryl group in the recognition capabilities of 2-aminopyridines towards carboxylic acids.
}

\author{
Miguel Gallegos · Sara Gil-Guerrero · A. Fernández-Alarcón · Diego \\ Bouzas-Ramos · Judith Martín · Carmen Concellón · Vicente del Amo · J. M. \\ Costa · R. Mendoza-Meroño · S. García-Granda · Ángel Martín Pendás · Aurora \\ Costales*
}

Received: date / Accepted: date

\begin{abstract}
Inspired by natural molecular recognition processes, many research efforts have been routed in recent years towards the design of new host-guest molecular systems based on non-covalent interactions. Within this field, 2-aminopyridines (2APs) have been widely studied due to their tunable spectroscopic response in presence of carboxylic acids. Herein, we present and analyze a novel family of 2AP core compounds based on 2phosphorylamidopyridine (2PAP). Linear response timedependent density functional theory (TD-DFT) has been used to characterize and model several spectroscopic properties of 2PAP. Our results, validated through experiments, show that TD-DFT can provide a reliable description of the electronic excited states of these aromatic systems. In addition, we have also studied the amino-imino tautomerization of 2AP and 2PAP under the light of TD-DFT tools. We show that the presence of a carboxylic acid has a catalytic effect on the tautomerization reaction, which otherwise does not occur spontaneously at room temperature. These results suggest that this low-cost computational approach can be applied to more complex organic systems derived from 2-aminopyridine, paving the way for the development of potentially useful sensing materials and organic species for molecular recognition.
\end{abstract}

Keywords Molecular recognition, amino-imino tautomerization, fluorescence, 2-aminopyridines

M. Gallegos, A. Fernández-Alarcón, D. Bouzas-Ramos, J. M. Costa, R. Mendoza, S. García-Granda, Á. Martín Pendás, A. Costales Department of Analytical and Physical Chemistry, University of Oviedo, E-33006, Oviedo, Spain.

E-mail: costalesmaria@uniovi.es

S. Gil-Guerrero

Department of Physical Chemistry, University of Vigo, E-36310, Vigo, Spain.

J. Martín, C. Concellón, V. del Amo

Department of Inorganic and Organic Chemistry, University of Oviedo, E-33006, Oviedo, Spain.

\section{Introduction}

Molecular recognition, the specific interaction between guest and host molecules via non-covalent interactions, ${ }^{1}$ plays a key role in many biological processes. Important examples include the pairing of nucleobases ${ }^{2,3}$ in deoxyribonucleic acid (DNA), standard enzyme-substrate recognition, antigen-antibody interactions and several steps in the neurotransmission pathway ${ }^{4,5}$. Inspired by these processes, the last years have witnessed sustained research efforts in the design of artificial systems with tailored properties in self-assembly ${ }^{6}$, host-guest chemistry, ${ }^{7,8}$ supramolecular chemistry ${ }^{9,10}$ and molecular docking. ${ }^{11} \mathrm{~A}-$ mong the different systems exhibiting this behavior, small organic molecular structure forming self-assembled structures or interacting with other molecules via hydrogen bonds (H-bond) have been thoroughly investigated. ${ }^{12-14}$

In this context, functionalized aromatic frameworks showing alternating H-bond donor-acceptor (D-A) patterns have attracted considerable interest as they can play an instrumental role in different biological processes, ${ }^{11,15,16}$ and 2-aminopyridines (2APs) 1 (Chart 1 ) have been the focus of an intense experimental ${ }^{17-20}$ and theoretical ${ }^{18,19}$ research over the years. From the molecular recognition point of view, 2APs of general form (1) (see Chart 1) endowed with $\mathrm{D}$ and $\mathrm{A}$ sites in their structure can recognize carboxylic acids 2 with a D-A pattern that mimics in a complementary way that of $2 \mathrm{AP}$, leading to the formation of the supramolecular complex [1.2] (Chart 2) stabilized via two H-bonds. Alternatively, a proton transfer reaction can also take place resulting in the formation of the $\left[\mathbf{1}^{+} \cdot \mathbf{2}^{-}\right]$complex, the relative yield of both processes depending on the specific molecular structure of the 2AP and on the experimental conditions. Additionally, 2APs have interesting spectroscopic features, in particular their medium or high fluorescence ${ }^{21}$ in a wide variety of experimental conditions, or their chemical versatility and synthetic accessibility, ${ }^{22}$ which makes them ideal fluorophores for a large number of applications. ${ }^{23}$ The fluorescence properties of the 2-aminopyridine core have been known for many decades, and hence they have been widely exploited for analytical and sensing purpo- 


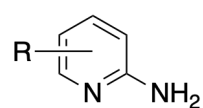

1

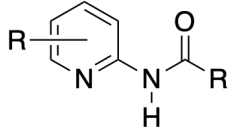

3

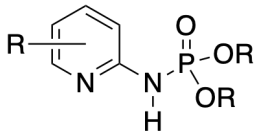

4
Chart 1 General structure of 2-aminopyridine and its amido and phosphoryl derivatives

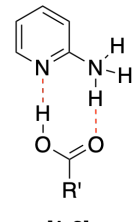

$[1 \cdot 2]$

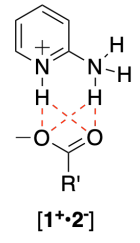

$$
\overbrace{\mathrm{R}^{1}}
$$

Chart 2 2-aminopyridine-carboxylic acid complexes illustrating the different stabilization mechanisms and structural modifications of the 2-aminopyridine core aimed at increasing the binding energy of these complexes.

ses. ${ }^{24,25}$ Of particular relevance is the modulation and flexibility of the spectroscopic properties of 2AP in the presence of carboxylic acids.

Aiming at optimizing the binding process of [1.2] complexes, Hamilton and others ${ }^{26-28}$ resorted to structural modification of the 2AP core and replaced the amino unit of 2APs by a carboxamide moiety. The resulting amidopyridines 3 (Chart 1 ), bearing a considerably more acidic proton at the amide group, are significantly more prone to participate in H-bonding phenomena. As a consequence, supramolecular complexes [3.2] (Chart 2) display enhanced binding constants ( $\mathrm{K}=100-200 \mathrm{M}^{-1}$ at $\left.298 \mathrm{~K}\right)$ in non-competitive organic solvents.

In order to increase the versatility and to enhance such modulation capabilities, we have synthesized for the first time a new family of 2AP derivatives 4 by introducing a phosphoryl group in the 2AP core $\mathbf{1}$ (Chart 1 ). Such chemical modification enhanced the spectroscopic properties of these new derivatives with respect to their simpler analogs. Motivated by these results, here we investigate the spectroscopic properties and the H-bonding ability of the structurally related 2PAP towards carboxylic acids. Early observations on this work have revealed that 2PAP is remarkably fluorescent, with a significantly larger change in quantum yield in the presence of a carboxylic acid than in the $\mathbf{1}$ or $\mathbf{3}$ counterparts. This, together with the existence of cheap and easy protocols for its synthesis and functionalization suggest that this family of compounds has a great potential to increase the molecular recognition capability of the 2APs. As a characteristic feature of this family, we use the bare 6-methyl-2-phosphorylamidopyridine (6Me-2PAP, see Fig. 1) and explore its complexation with formic acid to show the change in the spectroscopic properties of this kind of compounds. We have thus studied the impact of the presence of the phosphoryl group on the amino-imino tautomerization process. In addition, and following the previous trends found in already reported studies with the 2AP analogs ${ }^{29}$, we also address the impact that methyl substitution has on the spectroscopic properties of the 2PAP systems.

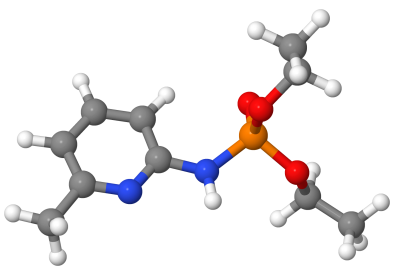

Fig. 1 Molecular structure of the 6-methyl-2-phosphorylamidopyridine investigated in this work, computed at B3LYP/aug-cc-pvdz.

The article is organized as follows. First, we briefly present the basic features of the experimental fluorescence spectra of 2APs and their modification upon phosphorylation. The computational methodology used to rationalize them is then presented, starting with validation strategies in the simplest 2AP case. Once a compromise is chosen, we turn to analyze the effect of methyl substitution in the pyridine ring on the spectrum and continue by showing our results on a representative member of the 2PAP family. Finally, we propose an explanation of the effect of the phosphoryl group on the molecular recognizing abilities of the 2AP core compounds by considering the reaction path of the amino-imino tautumerization of $2 \mathrm{AP}$ and $6 \mathrm{Me}-2 \mathrm{PAP}$ in the presence/absence of formic acid. To conclude, we summarize the main conclusions and the outlook of this work.

\section{Fluorescence of 2APs and 2PAPs.}

The two most important properties that a host-guest complex useful for molecular recognition purposes should display are: (i) a reasonable stability of the formed complex; (ii) the existence of significant changes in a given measurable property between the isolated host and guest and the host-guest association. It is well known that the UVVIS spectrum of 2APs displays a maximum of emission at $\lambda=330 \mathrm{~nm}^{1}$. The position and intensity of this band suffers a substantial change in the presence of carboxylic acids. For instance, Figure 2 shows the the experimental emission spectra of 6Me-2AP together with its change upon addition of a 1:10 formic acid solution. The intensity of the original emission band at $330 \mathrm{~nm}$ decreases as formic acid is added and a new emission band centered at $404 \mathrm{~nm}$ appears. Phosphorylation significantly enhances the fluorescence. This is shown in the newly synthesized 6Me-2PAP compound. It is important to notice that the position of the the bands is not widely affected by phosphorilation, but that the sensitivity of the 6Me-2PAP spectrum to the presence of formic acid increases drastically. The large observed difference in the spectroscopic behaviour between the original 2AP and the novel family of 2-phospoamidopyridine compounds herein presented has motivated us to study these properties of 2PAP family members. 


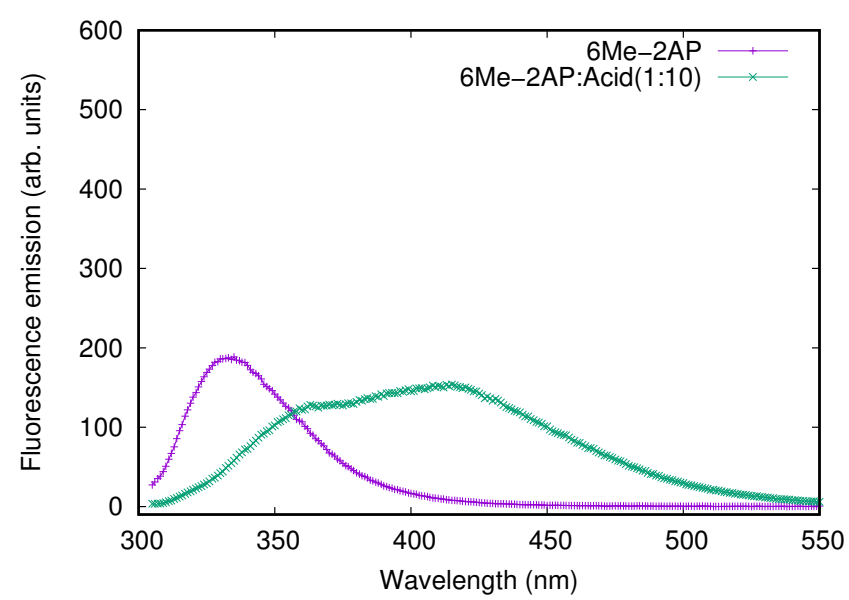

a) Previous work

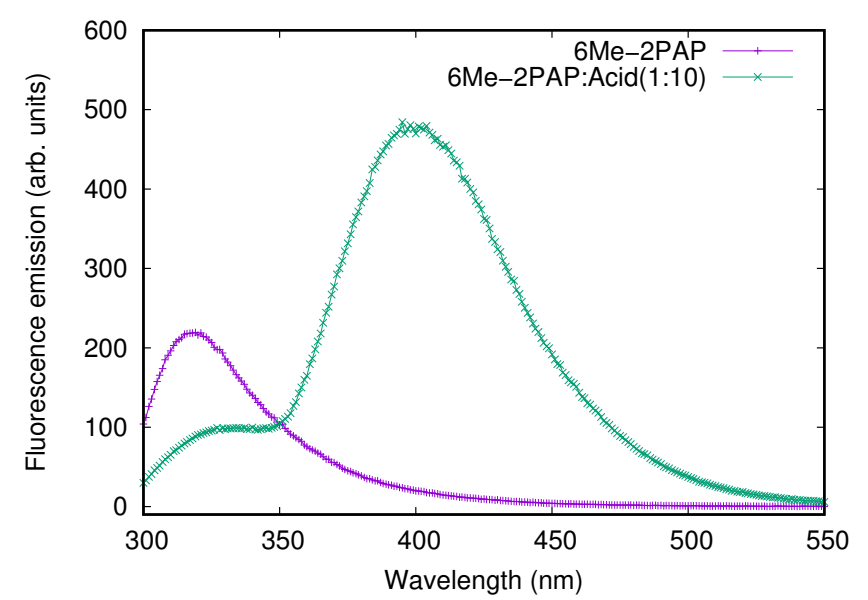

b) Our work

Fig. 2 Comparison of the experimental fluorescence spectra of 6Me-2AP (an already reported system ${ }^{29}$ ) and $6 \mathrm{Me}-2 \mathrm{PAP}$ (our novel compound) in the absence and presence of 10 equivalents of formic acid in dichloromethane solution.

Table 1 Computed transition frequencies and oscillator strengths for the absorption $\left(f_{a b s}\right)$ and for the emission $\left(f_{\text {ems }}\right)$ of 6Me-2AP with a set of density functionals. Calculations are reported with the cc-pvdz and aug-cc-pvdz basis sets in dichloromethane.

\begin{tabular}{lccccc}
\hline Functional & Basis set & $f_{a b s}$ & $f_{\text {ems }}$ & $\lambda_{\text {abs }}(\mathrm{nm} / \mathrm{eV})$ & $\lambda_{\text {ems }}(\mathrm{nm} / \mathrm{eV})$ \\
\hline B3LYP & cc-pvdz & 0.107 & 0.136 & $267 / 4.64$ & $306 / 4.05$ \\
B3LYP & aug-cc-pvdz & 0.114 & 0.150 & $275 / 4.51$ & $312 / 3.97$ \\
CAMB3LYP & cc-pvdz & 0.134 & 0.177 & $253 / 4.90$ & $289 / 4.30$ \\
CAMB3LYP & aug-cc-pvdz & 0.141 & 0.186 & $261 / 4.75$ & $297 / 4.17$ \\
HSE & cc-pvdz & 0.115 & 0.147 & $262 / 4.73$ & $300 / 4.13$ \\
HSE & aug-cc-pvdz & 0.120 & 0.159 & $269 / 4.61$ & $306 / 4.05$ \\
WB97X & cc-pvdz & 0.144 & 0.190 & $249 / 4.98$ & $285 / 4.35$ \\
WB97X & aug-cc-pvdz & 0.152 & 0.201 & $256 / 4.84$ & $294 / 4.22$ \\
WB97XD & cc-pvdz & 0.132 & 0.174 & $254 / 4.88$ & $290 / 4.28$ \\
WB97XD & aug-cc-pvdz & 0.140 & 0.186 & $261 / 4.75$ & $297 / 4.17$ \\
M062X & cc-pvdz & 0.137 & 0.181 & $250 / 4.96$ & $287 / 4.32$ \\
M062X & aug-cc-pvdz & 0.142 & 0.188 & $259 / 4.79$ & $297 / 4.17$ \\
\hline
\end{tabular}

\section{Computational methodology}

We have used a simple computational approach based on density functional theory (DFT) together with an implicit solvation model to account for solvent effects. Specifically, we have calculated the ground state equilibrium structures of 2PAP and its methyl derivatives at the DFT level of theory employing the B3LYP ${ }^{30-32}$ hybrid exchangecorrelation functional together with the aug-cc-pVDZ basis set. ${ }^{33}$ The vertical excitation energies of the lowestlying singlet excited states of interest were calculated using linear response (LR) TD-DFT, ${ }^{34}$ employing the same correlation-exchange functional and basis set. Solvent effects were incorporated using the continuum polarization model (PCM) approach ${ }^{35,36}$ using $\mathrm{CH}_{2} \mathrm{Cl}_{2}$ as solvent in the manner implemented in the Gaussian09 program. ${ }^{37}$ Equilibrium and non-equilibrium solvent effects were computed when appropriate employing the conventional linear response and state-specific solvation formalism. ${ }^{38-41}$ Using this calculation setup, both the ground and the lowest-lying singlet excited state equilibrium structures of 2PAP's were obtained and frequency calculations were performed to verify that the geometries found indeed correspond to a minimum of the potential energy hypersurface. Finally, the amino/imino tautomerization

\footnotetext{
$1200 \mu \mathrm{M}$ in a DCM solution at room temperature.
}

reaction in $6 \mathrm{Me}-2 \mathrm{PAP}$ was studied without and with the assistance of formic acid using the same computational approach.

In order to select an appropriate density functional to be used in the computational study of 2PAP, the spectroscopic properties of a reference system, 6-methyl-2aminopyridine (6Me-2AP), were evaluated with a series of functionals at the TD-DFT level of theory.

Table 1 collects the predicted absorption and emission maxima (in $\mathrm{nm} / \mathrm{eV}$ ) of $6 \mathrm{Me}-2 \mathrm{AP}$ in a dichloromethane solution employing the cc-pVDZ and aug-cc-pVDZ basis set. The results suggest that among the studied functionals, B3LYP is the one that offers the best results in comparison with the experimental data: the values of the experimental absorption and emission wavelength maxima are found at 287 and $333 \mathrm{~nm}$, respectively (see the ESI, section 4 for more details). We have thus chosen B3LYP in the following. Furthermore, the introduction of diffuse basis functions (aug-cc-pVDZ) in combination with the B3LYP functional yield the best overall results ( $\lambda=275 \mathrm{~nm}$ for absorption and $312 \mathrm{~nm}$ for emission). Based on these findings, we considered the B3LYP/augcc-pVDZ level of theory as the optimal one to be employed within this study.

All the theoretical results presented have been backed up by experiments. In the supporting information we col- 
Table 2 Experimental values of the fluorescence quantum yield $(\Phi)$ and calculated oscillator strengths for absorption $\left(f_{a b s}\right)$ and emission $\left(f_{e m s}\right)$. Absorption $\left(\lambda_{\text {abs }}\right)$ and emission $\left(\lambda_{\text {ems }}\right)$ wavelengths (in $\mathrm{nm}$ ) for the studied family of 2PAP (see the ESI Figures S10 to S13 for more details).

\begin{tabular}{lccccc}
\hline Molecule & $f_{\text {abs }}$ & $f_{\text {ems }}$ & $\Phi$ & $\lambda_{\text {abs }}$ & $\lambda_{\text {ems }}$ \\
\hline 2PAP & 0.105 & 0.146 & $7 \%$ & 256 & 293 \\
3Me-2PAP & 0.145 & 0.185 & $9 \%$ & 251 & 304 \\
4Me-2PAP & 0.084 & 0.120 & $7 \%$ & 259 & 292 \\
5Me-2PAP & 0.115 & 0.159 & $18 \%$ & 268 & 304 \\
6Me-2PAP & 0.150 & 0.197 & $16 \%$ & 261 & 295
\end{tabular}

lect a number of details on the synthesis (see the ESI section 10) and characterization of 2PAP compounds such as their X-ray structures, or their NMR and mass spectra (see the ESI section 2 and section 11, respectively). We also address the absorption and emission spectra of selected 2PAP and 2AP systems.

\section{Results and Discussion}

\subsection{Validation of the methodology}

The effect of the methyl perturbation in the spectroscopic properties of the phosphorylamidopyridine skeleton was evaluated through TD-DFT calculations as indicated previously.

As shown in Table 2, the position of the methyl group within the aromatic ring leads to significant changes in the predicted oscillator strengths for both the emission and the absorption process evaluated at the B3LYP/augcc-pVDZ level of theory. The introduction of a methyl group at position 4 leads to a decrease in the oscillator strengths, whereas, on the other hand, the presence of methyl groups in positions 3, 5 and 6 results in a net enhancement of the predicted oscillator strengths. These results, being in good agreement with experimental findings (see the ESI section 4 for more details), follow the already reported trends with the $2 \mathrm{AP}$ analogs, ${ }^{29,42,43}$ suggesting that the presence of the phosphoryl group does not significantly modify the way in which the methyl substitution of the aromatic ring affects the observed trend in the spectroscopic properties of the methyl substituted 2PAP derivatives.

However, the fluorescence quantum yield of 5Me-2PAP has been found to deviate significantly from this trend. Such deviation has already been previously described employing other computational methods in analogous systems. ${ }^{29,43}$

\subsection{Characterization of the spectroscopic properties}

The relative energy, oscillator strengths and electronic character of the four lowest-lying singlet excited states of $6 \mathrm{Me}-2 \mathrm{PAP}$ in $\mathrm{CH}_{2} \mathrm{Cl}_{2}$ computed as detailed above are collected in Table 3.

The maximum of the steady-state experimental lowest energy absorption band lies at $281 \mathrm{~nm},(4.41 \mathrm{eV})$
Table 3 Vertical excitation energies ${ }^{\mathrm{a}}(\Delta \mathrm{E}, \mathrm{nm})$, oscillator strength $(f)$ and character of the states ${ }^{b}$ of the four lowest-lying singlet excited states of $6 \mathrm{Me}-2 \mathrm{PAP}$ in $\mathrm{CH}_{2} \mathrm{Cl}_{2}$ calculated at the LR-TDB3LYP/aug-cc-pVDZ level of theory.

\begin{tabular}{|c|c|c|c|}
\hline State & $\Delta \mathrm{E}$ & $f$ & Transition \\
\hline $\mathrm{S}_{1}$ & $261(281)$ & 0.152 & ${ }^{1}\left(\pi, \pi^{*}\right)$ HOMO-LUMO \\
\hline $\mathrm{S}_{2}$ & $238-$ & 0.004 & ${ }^{1}\left(\mathrm{n}, \pi^{*}\right)$ HOMO-1-LUMO \\
\hline $\mathrm{S}_{3}$ & $226-$ & 0.044 & mixed $^{c}$ HOMO-LUMO+2 \\
\hline $\mathrm{S}_{4}$ & $224(234)$ & 0.212 & ${ }^{1}\left(\pi, \pi^{*}\right)$ HOMO-LUMO+1 \\
\hline
\end{tabular}

and compares reasonably well with the calculated vertical excitation to the optically allowed lowest-lying $S_{1}$ state $(261 \mathrm{~nm}, 4.74 \mathrm{eV})$, see Fig. 3 . This is a ${ }^{1}\left(\pi, \pi^{*}\right)$ state mostly characterized by a HOMO-LUMO single excitation (see Fig. 4) which is essentially localized at the 2AP core. The second experimental absorption band located at $234 \mathrm{~nm},(5.30 \mathrm{eV})$ also shows a reasonable agreement with the vertical excitation energy calculated for the second lowest-lying absorbing excited state, $\mathrm{S}_{4}$. This is also a ${ }^{1}\left(\pi, \pi^{*}\right)$ state and lies at $224 \mathrm{~nm}(5.54 \mathrm{eV})$ above the ground state. Like $S_{1}, S_{4}$ also exhibits a high degree of localization, although the latter is slightly more delocalized than $S_{1}$, with some contributions existing at the $\mathrm{P}$ atom as the most important single excitation contribution involving the HOMO and LUMO+1 orbitals (see Fig. 4). Between these states there are two less bright ones: $S_{2}$ with ${ }^{1}\left(\mathrm{n}, \pi^{*}\right)$ character, and $\mathrm{S}_{3}$ a mixed state that exhibits mainly diffuse (Rydberg) character but that also has contributions of a valence HOMO-LUMO+1 excitation. From the results obtained, it can be noticed that the energies of the bright excited states considered in the simulations are overestimated with respect to the experimental values, a fact that we ascribe to the well known limitations of the PCM.

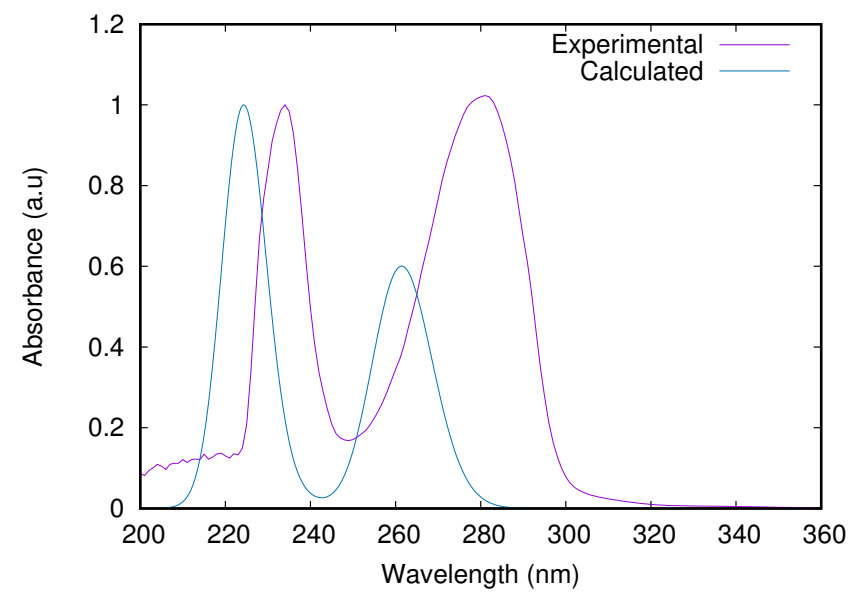

Fig. 3 LR-TD-B3LYP/aug-cc-pVDZ simulated absorption and experimental spectra for the $6 \mathrm{Me}-2 \mathrm{PAP}$ in $\mathrm{CH}_{2} \mathrm{Cl}_{2}$. (See the ESI section 1.5 for details to obtain the theoretical spectrum).

A comparison of the results obtained for 6Me-2PAP with those found for 2AP and 6Me-2AP (see the ESI, section 3 ) shows that the vertical excitation energies of the two lowest-lying absorbing excited states of $6 \mathrm{Me}-2 \mathrm{PAP}$ 


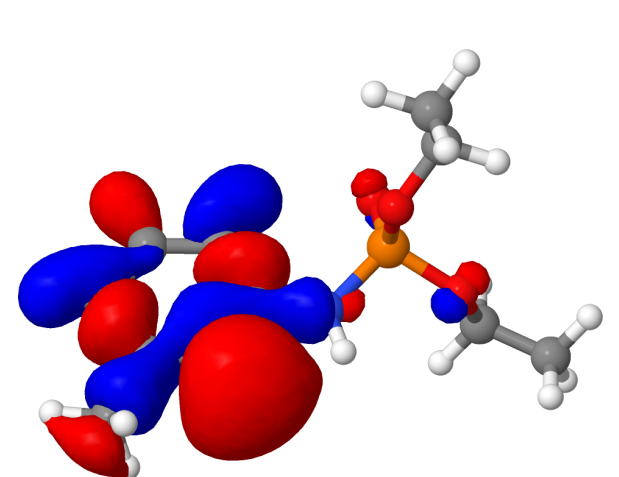

HOMO-1

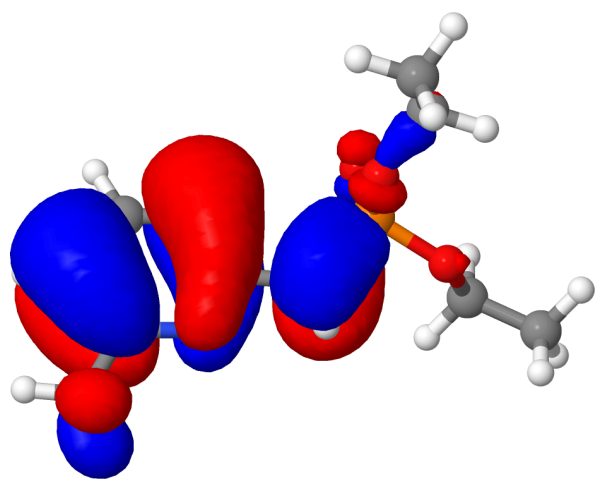

HOMO

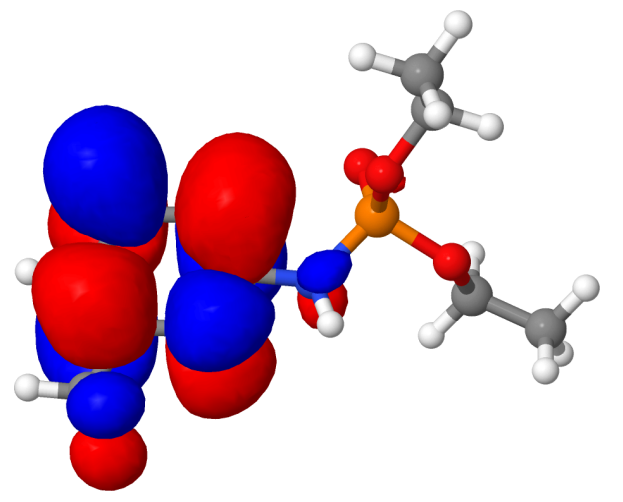

LUMO

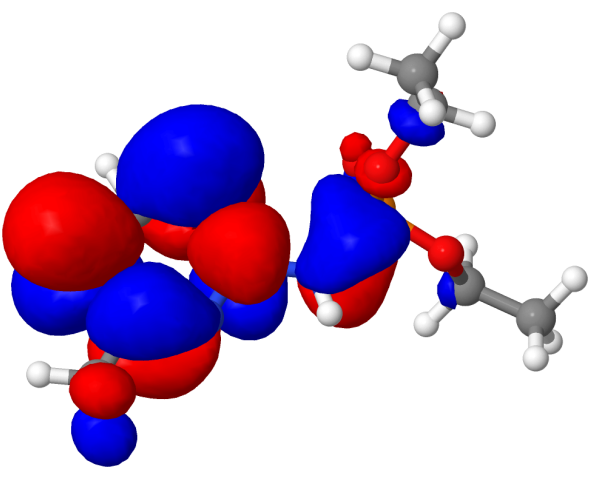

$\mathrm{LUMO}+1$

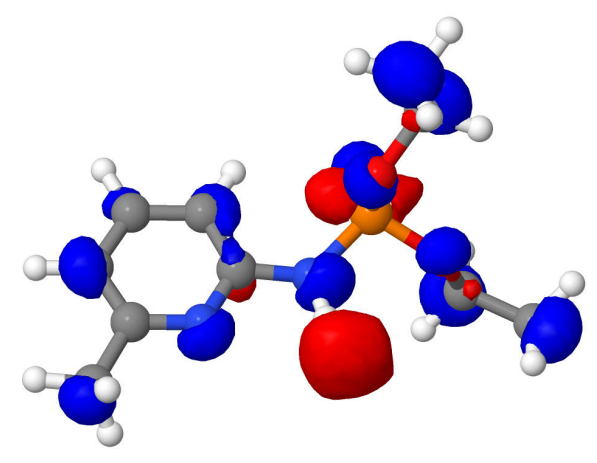

$\mathrm{LUMO}+2$

Fig. 4 Main frontier molecular orbitals of 6Me-2PAP. Rendered with Jmol, isovalue=0.02 a.u.

are very similar to those obtained for $2 \mathrm{AP}$, with the largest difference found for the high energy absorption band $\left(\mathrm{S}_{4}\right.$, $\sim 0.18 \mathrm{eV}$ ). In addition, the character of the states does not change significantly. The main differences between the two systems are an increase in the relative emission intensity of $\mathrm{S}_{1}$ in $6 \mathrm{Me}-2 \mathrm{PAP}$ as compared to that found in 2AP and the appearance in 2PAP of an extra dark state near degenerate with the higher lying absorbing state $\left(\mathrm{S}_{4}\right)$.

These results point out that, despite the evident increase in the oscillator strengths, the addition of the PO$\left(\mathrm{OCH}_{2} \mathrm{CH}_{3}\right)_{2}$ group to $2 \mathrm{AP}$ does not have a strong impact on the fundamental spectroscopic properties of the system, which very closely resemble those of the 2AP core structure. This is further substantiated if we consider the emission properties of 6Me-2PAP and 2AP. However, we have found that the computed emission energy at the $S_{1}$ equilibrium structure of 6Me-2PAP $(295 \mathrm{~nm}, 4.21 \mathrm{eV})$ agrees reasonably well with that of $6 \mathrm{Me}-2 \mathrm{AP}(312 \mathrm{~nm}$, $3.98 \mathrm{eV}$ ) being the nature of the emitting state the same $\left.{ }^{1}\left(\pi, \pi^{*}\right)\right)$. Furthermore, the main structural relaxation process responsible for the Stokes shift is, in both cases, the extension of the delocalization of the $\pi$-like orbitals involved in the de-excitation process. This is the result of structural changes mainly associated to the amino group (that adopts a planar conformation in the $S_{1}$ equilibrium structure instead of the pyramidalized one in the ground state equilibrium structure, in agreement with previous results ${ }^{23}$ ).

Both cases, 6Me-2AP and 6Me-2PAP, exhibit two absorption maxima. When they are excited at any of those, the same fluorescence emission maximum is obtained (see the ESI, Figs. S5 and S7) within the near UV region at 335 $\mathrm{nm}$ in agreement with Kasha's rule ${ }^{44}$. Following the previously mentioned procedure, we carried out the same study in the presence of formic acid and an equivalent behaviour is observed as shown in Figs. S5 and S7. The effect of formic acid will be properly discussed in the next section. Note that the calculated $S_{1}$ energy is overestimated due to several theoretical shortcomings.

\subsection{The carboxylic acid assisted proton transfer reaction}

The effect of the hydrogen bond assistance of simple carboxylic acids, such as formic acid, in the tautomerization reaction of the 2AP framework within the 6Me-2PAP scaffold has been studied by both experimental and theoretical means.

Experimentally (see the ESI Figure S5), upon the addition of formic acid the intensity of the main emission band of 6Me-2PAP (316 nm) is significantly decreased while a new emission band $(390 \mathrm{~nm})$ associated with the formation of the host-guest complex appears. In contrast, the absorption spectrum (see the ESI Figure S4) is not significantly perturbed by the addition of such species.

In order to explain the previously mentioned experimental findings, the effect of the carboxylic acid assistance of formic acid in the amino-imino tautomerization of the 2AP framework within the 6Me-2PAP system, and hence its impact in the overall spectroscopic properties, has been studied by means of TD-DFT calculations. The results reveal that, analogously to what it has been already reported for the $2 \mathrm{AP}$ system ${ }^{45-48}$, the spontaneous 
tautomerization of 6Me-2PAP (see Figure 5c) is not likely to occur in the ground state as reflected by a reasonably large activation energy of $39 \mathrm{kcal} / \mathrm{mol}$. The transition state associated with this process is shown in Figure 6a.

Regarding the stability of the amino-imino tautomers (in the absence of formic acid) of the 6Me-2PAP system, the amino species is more stable than the imino one by $3.91 \mathrm{kcal} / \mathrm{mol}$. This result significantly differs from that observed for their 2AP analogs (see the ESI section 8), with a mean difference in energy between both tautomers of $11.48 \mathrm{kcal} / \mathrm{mol}$. The higher stability of the amino form can be attributed to the aromatic character of the pyridinic ring, which otherwise is partially disturbed in the imino species. Similarly, the difference in energy between both species $2 \mathrm{AP}$ and $6 \mathrm{Me}-2 \mathrm{PAP}$ indicates that though in both cases the amino form is significantly more stable than the imino one, the presence of the phosphoryl group leads to a relative stabilization of the imino tautomer with respect to the amino one. Such result could be rationalized by considering the ability of the phosphoryl moiety to partially delocalize electron density of the heterocyclic ring in the imino form, leading to a highly delocalized conjugated system. Analogously, the activation energy for the intramolecular proton transfer reaction in the presence of the phosphoryl group is significantly decreased for the non-assisted tautomerization by a total of $10 \mathrm{kcal} / \mathrm{mol}$ with respect to their simpler analogs (2AP). This also suggests that though the spontaneous intramolecular proton transfer reaction of the 6Me-2PAP system is unlikely to occur at room temperature, the existence of the phosphoryl moiety in the general pyridinic skeleton favors such tautomerization process. Considering the late transition state character of the activated complex involved in this transformation, being closer to the geometry of the product, this catalytic effect could be rationalized by accounting for the previously mentioned stabilization of the imino form in the presence of the phosphoryl group.

On the other hand, the presence of formic acid induces dramatic changes in the chemical transformation under scrutiny. Initially, a 6Me-2PAP-formic acid HB assisted supramolecular complex (amino form) is produced, as shown in Figure 7. Such species can easily lead, via single proton transfer reaction, to an ionic carboxylatepyridinium species (see Figure 7 a) and d) for $S_{0}$ and $S_{1}$, respectively). Similarly, this intermediate can experience with ease an additional proton transfer reaction yielding the corresponding 6Me-2PAP (imino)-formic acid HB assisted supramolecular complex (see Fig. 7 b) and e) for $S_{0}$ and $S_{1}$, respectively). The activation energies for both, the former and the later proton transfer reactions, are $1.19 \mathrm{kcal} / \mathrm{mol}$ and $3.5 \mathrm{kcal} / \mathrm{mol}$ respectively (transition states are shown in Figure 6), indicating that the presence of formic acid reduces the overall barrier by a factor of 10 with respect to the non-catalyzed reaction. These results, following a similar trend to that reported for 2aminopyridines, ${ }^{49-51}$ clearly evidence the catalytic effect of simple carboxylic acids in the amino-imino tautomerization reaction of $6 \mathrm{Me}-2 \mathrm{PAP}$.
Table 4 Calculated absorption $\left(\lambda_{\mathrm{abs}}\right)$ and emission $\left(\lambda_{\mathrm{ems}}\right)$ maxima and computed oscillator strengths $\left(f_{a b s}\right),\left(f_{e m s}\right)$ for the different 6Me-2PAP-formic acid supramolecular complexes.

\begin{tabular}{lcccc}
\hline Supramolecular complex & $f_{\text {abs }}$ & $f_{\text {ems }}$ & $\lambda_{\text {abs }}$ & $\lambda_{\text {ems }}$ \\
\hline Amino (HB) & 0.168 & 0.225 & 271 & 332 \\
Ionic & 0.213 & 0.225 & 284 & 331 \\
Imino (HB) & 0.210 & 0.182 & 304 & 370 \\
\hline
\end{tabular}

The results follow a similar trend to those observed for their simpler analogs which also experience a net catalytic effect in the presence of formic acid. Conversely, a comparison of the reaction energy profiles of both systems reveals the different nature of the intermolecular proton transfer reaction. According to our study, in the case of 2AP (the IRC path is shown in Figs. S17, S18a, and S18b), a quasi-concerted double proton transfer reaction (with a mean activation energy of $6.85 \mathrm{kcal} / \mathrm{mol}$ ) is likely to occur in the presence of formic acid. These findings differ strongly from those obtained for 6Me-2PAP, in which two non-concerted single proton transfer reactions are likely to be responsible for the assisted intermolecular tautomerization. These observations indicate that the presence of the phosphoryl group does have an additional catalytic effect in the overall tautomerization, while also favoring the ionic form over the tautomeric ones in the presence of formic acid.

Similarly, in the presence of formic acid, 6Me-2PAP has a mean energy difference between the amino-imino tautomers of $1.8 \mathrm{kcal} / \mathrm{mol}$, a value which is significantly lower than the $7 \mathrm{kcal} / \mathrm{mol}$ found for the $2 \mathrm{AP}$ system (see the ESI section 8 for more details). Once again, this confirms the ability of the phosphoryl moiety to further stabilize the imino species with respect to the amino tautomer.

These theoretical findings are in agreement with the observed perturbation in the spectroscopic properties of 6Me-2PAP-formic acid system. According to both experimental (Fig. S4) and computational results collected in Table 4 (admitting an offset of $0.18 \mathrm{eV}$ ), the new emission band at $390 \mathrm{~nm}$ is associated with the the imino (HB) complex, proving that the spontaneous amino-imino equilibrium of the studied 6Me-2PAP systems can easily take place in the presence of formic acid. Furthermore, as shown in Figure 7 the amino HB species in the $S_{1}$ (Figure $7 \mathrm{f}$ ) state experiences the single proton transfer reaction leading to the carboxylate-pyridinium complex which is the same as the ionic form Fig. $7 \mathrm{~d}$ ), showing that such transformation could take place spontaneously in the first excited state.

Overall, our results stress the strong influence that the phosphoryl group has on the general amino-imino tautomerization of the 2AP core within the 2PAP derivatives, which ultimately favors the partial formation of both the ionic and the imino species up to a much higher extent than on their 2AP analogs. Such findings are in accordance with the experimental results, proving the superior capabilities of this system to recognize simple carboxylic acids. 


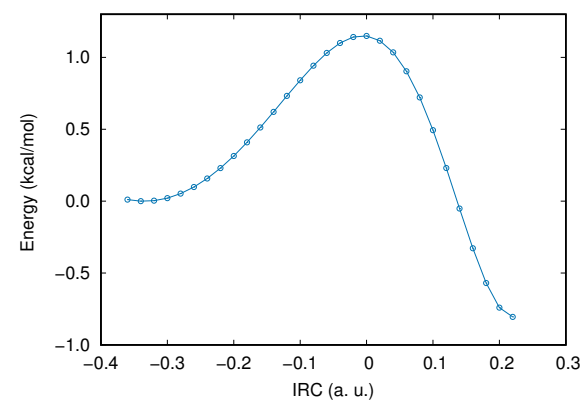

a) Catalyzed Amino-Ionic

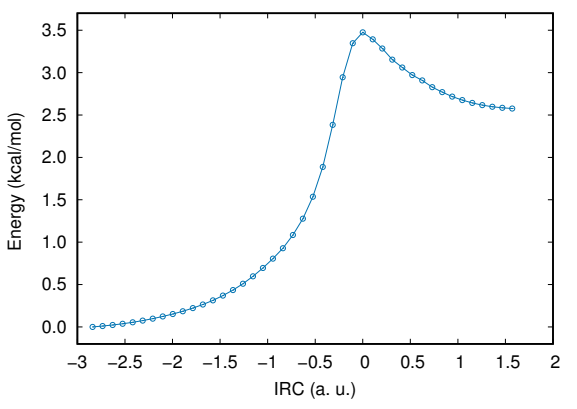

b) Catalyzed Ionic-Imino

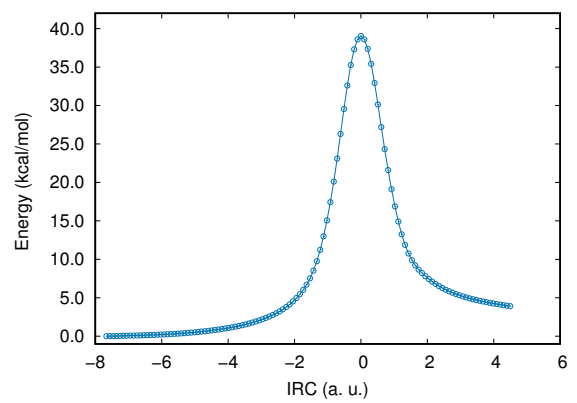

c) Non-catalyzed

Fig. 5 Reaction paths for the bare and catalyzed amino-imino tautomerization of 6Me-2PAP.

a)

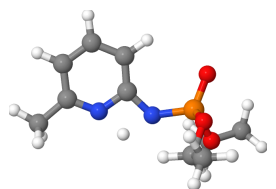

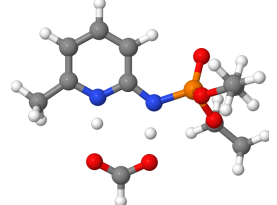

b)

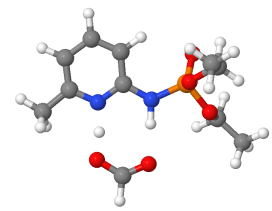

c)
Fig. 6 Transition states for the bare and catalyzed amino-imino tautomerization of 6Me-2PAP: a) Non-catalyzed Amino-Imino b) Catalyzed Salt-Imino and c) Catalyzed Amino-Salt. Rendered with Jmol.

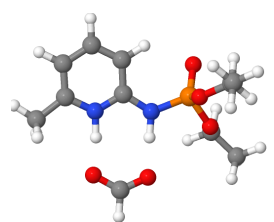

a) Ionic $S_{0}$

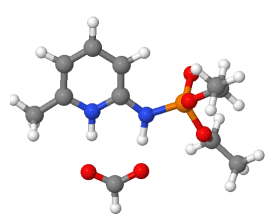

d) Ionic $S_{1}$

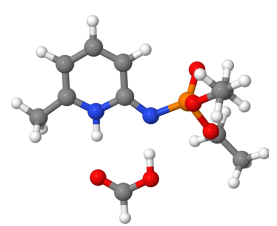

b) Imino $S_{0}$

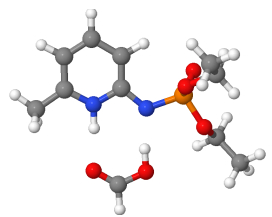

e) Imino $S_{1}$

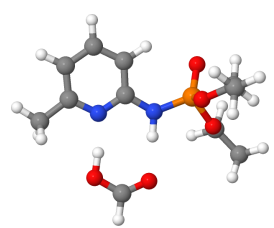

c) Amino $S_{0}$

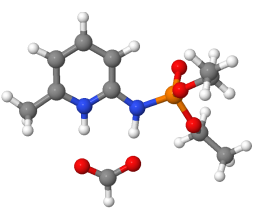

f) Amino $S_{1}$
Fig. 7 Fundamental state and first lowest-lying equilibrium geometries of the hydrogen bonded and ionic supramolecular complexes.

\section{Conclusion}

In this work we have studied how the introduction of a phosphoryl (PO) group in the general 2AP skeleton modifies its fluorescence spectrum. According to our computational study, in good agreement with experimental findings, the phosphoryl group does not significantly affect the absorption and emission maxima $\left(\lambda_{\max }\right)$ of the bare 2AP system. On the other hand, the presence of such a simple group leads to an enormous enhancement of the fluorescence quantum yield in the presence of formic acid. These findings indicate that such chemical modification does modify the spectroscopic properties of the "non-amino" forms of 2PAP as a consequence of a proton transfer reaction. Overall, the results obtained in this work point out the promising capabilities of 2PAP systems and their derivatives as more effective recognition and sensing systems for both simple carboxylic acids and possibly more complex moieties bearing D-A patterns.

\section{Acknowledgements}

We acknowledge financial support form Spanish MINECO/FEDER, grants CTQ2015-65790-P and CTQ2016-76829$\mathrm{R}$ and Principado de Asturias Government grant FC-GRUPINIDI/2018/000117. C. C. expresses her gratitude to MINECO for the award of "Ramón y Cajal" contract (RYC-201416021). AFA thanks to CONACyT/México for his scholarship 436154. We also thank Dr. P. Braña for fruitful discussions.

\section{References}

1. D. Uhlenheuer, K. Petkau, L. Brunsveld, Chem. Soc. Rev. 39(8), 2817 (2010)

2. M.J. Hannon, Chem. Soc. Rev. 36, 280 (2007)

3. P. B. Dervan, Biooorg. Med. Chem. 9, 2215 (2001). DOI 10.1016/S0968-0896(01)00262-0

4. S. Kruss, M.P. Landry, E. Vander Ende, B.M. Lima, N.F. Reuel, J. Zhang, J. Nelson, B. Mu, A. Hilmer, M. Strano, J. Am. Chem. Soc. 136(2), 713 (2014)

5. M. Maness, Patricia F; Schachner, Nat. Neurosci. 10, 19 (2007)

6. A. Zafar, S.J. Gib, Y. Hamuro, A.J. Carr, A.D. Hamilton, Tetrahedron 56, 8419 (2000)

7. J. Zhou, G. Yu, F. Huang, Chem. Soc. Rev. 46, 7021 (2017)

8. G. Yu, K. Jie, F. Huang, Chem. Rev. 115(15), 7240 (2015)

9. A.G. Slater, L.M.A. Perdigão, P.H. Beton, N.R. Champness, Acc. Chem. Res. 47(12), 3417 (2014)

10. M. kit Leung, A.B. Mandal, C.C. Wang, G.H. Lee, S.M. Peng, H.L. Cheng, G.R. Her, I. Chao, H.F. Lu, Y.C. Sun, M.Y. Shiao, P.T. Chou, J. Am. Chem. Soc. 124(16), 4287 (2002)

11. N.A. Caballero, F.J. Meléndez, A. Niño, C. MuñozCaro, J. Mol. Model. 13(5), 579 (2007)

12. H.J. Schneider, Chem. Soc. Rev. 23, 227 (1994)

13. Y. Kato, M.M. Conn, J. Rebek, Proc. Natl. Acad. Sci. USA 92(4), 1208 (1995)

14. D.M. Perreault, X. Chen, E.V. Anslyn, Tetrahedron 51(2), 353 (1995). Molecular Recognition

15. S. Mukherjee, S. Majumdar, D. Bhattacharyya, J. Phys. Chem. B 109(20), 10484 (2005)

16. I. Wickelgren, Science 297, 178 (2002) 
17. A. Weisstuch, A.C. Testa, J. Phys. Chem. 72(6), 1982 (1968)

18. T. Schultz, E. Samoylova, W. Radloff, I.V. Hertel, A.L. Sobolewski, W. Domcke, Science 306, 1765 (2004)

19. R. Wu, P. Nachtigall, B. Brutschy, Phys. Chem. Chem. Phys. 6, 515 (2004)

20. J.A. Farmer, C.T. Campbell, L. Xu, G. Henkelman, J. Am. Chem. Soc. 131(8), 3098 (2009)

21. R. Rusakowicz, A.C. Testa, J. Chem. Phys. 72(7), 2680 (1968)

22. A.T. Londregan, S. Jennings, L. Wei, Org. Lett. 12(22), 5254 (2010)

23. F. Zhang, Y.J. Ai, Y. Luo, W.H. Fang, J. Chem. Phys. 130(14), 144315 (2009)

24. R.R. Koner, S. Sinha, S. Kumar, C.K. Nandi, S. Ghosh, Tetrahedron Lett. 53(18), 2302 (2012)

25. T. Zengeya, P. Gupta, E. Rozners, Angew. Chem. Int. Ed. 51(50), 12593 (2012)

26. F. Garcia-Tellado, S. Goswami, S.K. Chang, S.J. Geib, A.D. Hamilton, J. Am. Chem. Soc. 112(20), 7393 (1990)

27. C. Vicent, E. Fan, A.D. Hamilton, Tetrahedron Lett. 33(30), 4269 (1992)

28. J. Yang, E. Fan, S.J. Geib, A.D. Hamilton, J. Am. Chem. Soc. 115(12), 5314 (1993)

29. A.C. Testa, U.P. Wild, J. Phys. Chem. 83(23), 3044 (1979)

30. L.W. S. H. Vosko, M. Nusair, Can. J. Phys. 58(8), 1200 (1980)

31. C. Lee, W. Yang, R.G. Parr, Phys. Rev. B 37(2), 785 (1988)

32. A.D. Becke, J. Chem. Phys. 98(7), 5648 (1993)

33. T.H. Dunning, J. Chem. Phys. 90(2), 1007 (1989)

34. U.C. A., Time-Dependent Density-Functional Theory: Concepts and Applications (Oxford Graduate Texts, 2011)

35. C.J. Cramer, D.G. Truhlar, Chem. Rev. 99(8), 2161 (1999)

36. J. Tomasi, B. Mennucci, R. Cammi, Chem. Rev. 105(8), 2999 (2005). PMID: 16092826

37. M.J. Frisch, G.W. Trucks, H.B. Schlegel, G.E. Scuseria, M.A. Robb, J.R. Cheeseman, G. Scalmani, V. Barone, B. Mennucci, G.A. Petersson, H. Nakatsuji, M. Caricato, X. Li, H.P. Hratchian, A.F. Izmaylov, J. Bloino, G. Zheng, J.L. Sonnenberg, M. Hada, M. Ehara, K. Toyota, R. Fukuda, J. Hasegawa, M. Ishida, T. Nakajima, Y. Honda, O. Kitao, H. Nakai, T. Vreven, J.A. Montgomery, Jr., J.E. Peralta, F. Ogliaro, M. Bearpark, J.J. Heyd, E. Brothers, K.N. Kudin, V.N. Staroverov, R. Kobayashi, J. Normand, K. Raghavachari, A. Rendell, J.C. Burant, S.S. Iyengar, J. Tomasi, M. Cossi, N. Rega, J.M. Millam, M. Klene, J.E. Knox, J.B. Cross, V. Bakken, C. Adamo, J. Jaramillo, R. Gomperts, R.E. Stratmann, O. Yazyev, A.J. Austin, R. Cammi, C. Pomelli, J.W. Ochterski, R.L. Martin, K. Morokuma, V.G. Zakrzewski, G.A. Voth, P. Salvador, J.J. Dannenberg, S. Dapprich, A.D. Daniels, ÃČ. Farkas, J.B. Foresman, J.V. Ortiz, J. Cioslowski, D.J. Fox. GaussianÃćÂĹÂij09 Revision
E.01. Gaussian Inc. Wallingford CT 2009

38. C. R., M. B., J. Chem. Phys. 110(20), 9877 (1999)

39. M. Cossi, G. Scalmani, N. Rega, V. Barone, J. Chem. Phys. 114, 5691 (2001)

40. T. Gustavsson, A. Bánász, E. Lazzarotto, D. Markovitsi, G. Scalmani, M.J. Frisch, V. Barone, R. Improta, J. Am. Chem. Soc. 128(2), 607 (2006)

41. R. Improta, V. Barone, S. F., Angew. Chem. Int. Ed. 46, 405 (2007)

42. R. Rautela, H. Joshi, N. Joshi, N. Tewari, S. Pant, J. Lumin. 132(8), 2151 (2012)

43. M.F.S. Khan, J. Wu, B. Liu, C. Cheng, M. Akbar, Y. Chai, A. Memon, R. Soc. Open Sci. 5(2), 171719 (2018)

44. M. Kasha, Faraday Discuss. pp. 14-19 (1950)

45. K. Inuzuka, A. Fujimoto, Bull. Chem. Soc. Jpn. 63, 971 (1990)

46. J. Konijnenberg, A. Herbert Huizer, C.A.G.O. Varma, J. Chem. Faraday Trans. 85(9), 1539 (1989)

47. T. Kitamura, A. Hikita, A. Ishikawa, H. Fujimoto, Spectrochimica Acta Part A 62, 1157 (2005)

48. S. Chai, G.J. Zhao, P. Song, S.Q. Yang, J.Y. Liu, K.L. Han, Phys. Chem. Chem. Phys. 11, 4385 (2009)

49. F.T. Hung, W.P. Hu, T.H. Li, C. Cheng, C. Pi-Tai, J. Phys. Chem. A 107, 3244 (2003)

50. J. Catalán, M. Carles del Valle, J. anda Kasha, Proc. Natl. Acad, Sci 96, 8338 (1999)

51. A. Douhal, M. Guallar, V. Moreno, J.M. Lluch, Chem. Phys. Lett. 256, 370 (1996) 